\title{
Quadriparesis after bariatric surgery: Case report
}

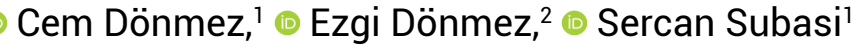 \\ 'Department of General Surgery, Muğla Sıtkı Koçman University Faculty of Medicine, Muğla, Turkey \\ ${ }^{2}$ Department of Anesthesiology and Reanimation, Yatagan Public Hospital, Muğla, Turkey
}

\begin{abstract}
Obesity is one of the most significant public health concerns of the $21^{\text {st }}$ century. The number of surgeries performed due to obesity continues to increase. Laparoscopic sleeve gastrectomy is the most commonly performed laparoscopic bariatric surgery and nutritional deficiencies after bariatric surgery are common. In weight-loss surgery patients with a nutritional deficiency, it should be kept in mind that the patient may have multiple vitamin deficiencies. Lifetime nutritional status follow-up is necessary. An appropriately detailed preoperative evaluation and postoperative care, as well as patient education, are essential to follow-up. There are guidelines available that recommend routine nutritional supplementation for the treatment and prevention of deficiencies. The aim of this manuscript was to describe the clinical impact of the current evidence.
\end{abstract}

Keywords: Bariatric surgery; laparoscopic sleeve gastrectomy; nutritional deficiency; obesity.

\section{Introduction}

The rising problem of obesity is one of the most significant public health concerns of 21st century. WHO (World Health Organization) reported 400 million people are obese and described obesity as pandemic..$^{[1]}$

Clinical guidelines for the obesity management describes body mass index (BMI) of $25 \mathrm{~kg} / \mathrm{m}^{2}$ to $29.9 \mathrm{~kg} / \mathrm{m}^{2}$ as overweight and BMI of $\geq 30 \mathrm{~kg} / \mathrm{m}^{2}$ as obesity. A study in the United States between 2011-2012 showed that the prevalence of obesity was $34.9 \%$ in adults. ${ }^{[2]}$ Review of clinical trials demonstrated that bariatric surgery is an unrivalled treatment option for obesity patients with sustained longterm weight loss, decreasing co morbidities, and improvement of the quality of life and life expectancy. ${ }^{[3]}$

Bariatric surgery has different types and preservation of the entire small bowel reduces the risk of vitamin defi- ciencies. However, patients should apply to their appointments and follow-ups. Weight loss surgery patients should be educated about their nutrition status and schedule their appointments postoperative 1 to 2 weeks, at months $1,2,3,6$, and 9 after surgery, and then yearly. ${ }^{[4]}$ Their nutrition should be managed according to "American Society for Metabolic and Bariatric Surgery" guidelines.

\section{Case Report}

A 20 -year-old woman, class 3, BMI $43.4 \mathrm{~kg} / \mathrm{m}^{2}$ underwent laparoscopic sleeve gastrectomy in other center. The procedure was well tolerated without any complications. The patient lost $27 \mathrm{~kg}$. relative to her preoperative weight in 4 months period. She had never attended her visits and follow-ups postoperatively. Her daily oral food intake was normal without any alcoholic abuse. Four months after LSG surgery she was admitted to the Neurology 
Department with quadriparesis. She had numbness with tingling in the feet and her both legs, weakness in arm and soon later she was unable to walk alone. She was presumed Guillain-Barre syndrome at another hospital and treated with intravenous immunoglobulin for 5 days. However, despite this therapy, the patient didn't recover. Vital signs were normal. Magnetic resonance imaging of the brain and spine were subsequently performed and no pathological findings were reported. Lumbar puncture was performed with the following results: 3 leucocytes, 0 red cell, proteins $32.5 \mathrm{mg} / \mathrm{dL}$, and glucose $57 \mathrm{mg} / \mathrm{dL}$ (plasma glucose). Electromyogram (EMG) showed signs of sensory-motor polyneuropathy. Her Glasgow Coma Score was E4M6V5. Pupils were isocoric. Complete blood count, electrolytes, renal, and hepatic functions were normal. Infectious and inflammatory markers were negative. She had low-amplitude gaze-evoked nystagmus in left horizontal gaze. She had quadriparesis 3/5 in upper limbs and lower limbs. Deep tendon reflexes were absent. Babinski sign was negative. There were no pathological findings in her cerebellar examination. Haemoglobin was $13.3 \mathrm{~g} / \mathrm{dL}$, mean corpuscular volume $93 \mathrm{fL}$, folate level 1.5 (4.6-18.7) ng/mL, vitamin B12 was $874.7 \mathrm{pg} / \mathrm{mL}$ (197-771), ferritin 181.2 (13-150) ng/mL, vitamin D 16.37 (20-50) ng/ $\mathrm{ml}$, thiamine $29.5(25-75) \mu \mathrm{g} / \mathrm{L}$. The patient had folate deficiency. She was given $1000 \mu \mathrm{g}$ folic acid by mouth daily. We started thiamine administration $100 \mathrm{mg} /$ day. The polyneuropathy was responsive to folic acid and thiamine replacement therapy. In one week, the patient started to gain muscle strength and was able to walk alone. After 42 days she was discharged with full recovery.

\section{Discussion}

The pathophysiology of severe obesity and the physiological mechanisms after bariatric surgery are yet to be understood. Initially, bariatric surgery is categorized into restrictive (Laparoscopic gastric banding [LAGB] and laparoscopic sleeve gastrectomy [LSG]) and/or malabsorptive operations (Roux- en-Y gastric bypass [RYGB], biliopancreatic diversion [BPD], duodenal switch [DS]). Laparoscopic sleeve gastrectomy is the first step and it has been performed as a part of DS and gaining popularity in the US. Laparoscopic sleeve gastrectomy is considered a safe procedure due to a low risk of nutritional deficiencies and gastric bypass is associated with increased nutritional deficiencies risk profile due to its complexity and alteration of gastrointestinal anatomy. Laparoscopic sleeve gastrectomy has lower complication rates than
BPD/DS and RYGB and it is associated with fewer vitamin deficiencies and malnutrition.

This case highlights several important aspects of the neurological manifestations of bariatric surgery patients with vitamin deficiencies. Nutritional deficiencies after bariatric surgery are common. It has been reported in the literature that the bariatric surgery patients could develop vitamin B1 (Thiamine) and vitamin B9 (folic acid) deficiency and the patient could present with neurological disorders.

Thiamine deficiency has a complex pathophysiology encompassing nutritional, metabolic, enzymatic, and neuroinflammatory diseases. Some of the thiamine deficiency related diseases are dry beriberi, wet beriberi, and metabolic acidosis. Dry beriberi or 'acute nutritional polyneuropathy' associated with peripheral neurodegeneration imitates Guillain-Barré syndrome. It can occur typically with weakness, paraesthesia with rapid onset. ${ }^{[5]}$

Folate deficiency polyneuropathies could affect lower and upper extremities with sensorimotor loss. The absence of ankle jerks and impairment of vibration sense are some characteristics of folate deficiency polyneuropathies. ${ }^{[6]}$ These patients initially present with numbness $s$ in the distal portion of lower extremities. ${ }^{[7]}$

In weight loss surgery patients with nutritional deficiency, it should be kept in mind that the patient could have multiple vitamin deficiencies. Due to possible nutritional complications resulting from bariatric surgery, it is important to provide complex screening and prevention of nutritional deficiencies. Lifetime nutritional status follow-ups are necessary. Vitamin and mineral supplementation should begin after 2-4 days of surgery. There are guidelines available that recommends routine nutritional supplementation for treatment and prevention of deficiencies. ${ }^{[8]}$

Laparoscopic sleeve gastrectomy is the most commonly performed laparoscopic bariatric surgery. Nutritional deficiencies are more common after malabsorptive surgeries like BPD, RYGB operations than restrictive procedures (LSG and LAGB). ${ }^{[9]}$ In our case, the patient missed or didn't care her follow-ups. In a clinical report on nutritional and pharmacologic challenges after bariatric surgeries, Lizer et al. ${ }^{[10]}$ recommended that all these patients should receive lifelong vitamin and mineral supplementation postoperatively. However, there are also opinions that only RYGB cohort should receive this form of prevention. 
In conclusion, although LSG has lower complication and malnutrition rates than BPD and RYGB, pre-operative and post-operative B-12 assessment still should be done to all LSG patients. Lifelong vitamin and mineral supplementation is necessary. A multidisciplinary team approach is mandatory as dieticians play an essential role in the perioperative period. In summary in preoperative evaluation and postoperative care, patient education is essential. The bariatric multidisciplinary team should be created and surgeons must perform bariatric surgeries at Centers of Excellence (COE).

\section{Disclosures}

Informed Consent: Written informed consent was obtained from the patient about her datas will be used in scientific publication.

Peer-review: Externally peer-reviewed.

Conflict of Interest: None declared.

\section{References}

1. Obesity: preventing and managing the global epidemic. Report of a WHO consultation. World Health Organ Tech Rep Ser 2000;894:i-xii, 1-253.

2. Ogden $\mathrm{CL}$, Carroll MD, Kit BK, Flegal KM. Prevalence of childhood and adult obesity in the United States, 2011-2012. JAMA 2014;311:806-14. [CrossRef]
3. Boza C1, Salinas J, Salgado N, Pérez G, Raddatz A, Funke R, et al. Laparoscopic sleeve gastrectomy as a stand-alone procedure for morbid obesity: report of 1,000 cases and 3-year follow-up. Obes Surg 2012;22:866-71. [CrossRef]

4. Allied Health Sciences Section Ad Hoc Nutrition Committee, Aills L, Blankenship J, Buffington C, Furtado M, Parrott J. ASMBS allied health nutritional guidelines for the surgical weight loss patient. Surg Obes Relat Dis 2008;4:S73-108.

5. Abdou E, Hazell AS. Thiamine deficiency: an update of pathophysiologic mechanisms and future therapeutic considerations. Neurochem Res 2015;40:353-61. [CrossRef]

6. Botez MI, Peyronnard JM, Bachevalier J, Charron L. Polyneuropathy and Folate Deficiency. Arch Neurol 1978;35:581-4.

7. Koike H, Takahashi M, Ohyama K, Hashimoto R, Kawagashira $\mathrm{Y}$, lijima $\mathrm{M}$, et al. Clinicopathologic features of folate-deficiency neuropathy. Neurology 2015;84:1026-33. [CrossRef]

8. Mechanick JI, Youdim A, Jones DB, Garvey WT, Hurley DL, McMahon $\mathrm{M}$, et al. Clinical practice guidelines for the perioperative nutritional, metabolic, and nonsurgical support of the bariatric surgery patient-2013 update: cosponsored by American Association of Clinical Endocrinologists, The Obesity Society, and American Society for Metabolic \& Bariatric Surgery. Obesity (Silver Spring) 2013;21: S1-27. [CrossRef]

9. Kehagias I, Karamanakos SN, Argentou M, Kalfarentzos F. Randomized clinical trial of laparoscopic Roux-en-Y gastric bypass versus laparoscopic sleeve gastrectomy for the management of patients with $B M l<50 \mathrm{~kg} / \mathrm{m} 2$. Obes Surg 2011;21:1650-6. [CrossRef]

10. Lizer $\mathrm{MH}$, Papageorgeon $\mathrm{H}$, Glembot TM. Nutritional and pharmacologic challenges in the bariatric surgery patient. Obes Surg 2010;20:1654-9. [CrossRef] 